\title{
PROVIDER CREATED DEMAND AND COST OF TREATING NON-FATAL ROAD TRAFFIC INJURIES IN KERALA, INDIA
}

\author{
Dr. Godwin SK $1 \bowtie$
}

${ }^{1}$ Associate Professor, Department of Economics, Government College for Women, Thiruvananthapuram- 695 014, Kerala, India

Received 17 November 2021

Accepted 25 December 2021

Published 31 January 2022

\section{CorrespondingAuthor}

Dr. Godwin SK, godwinsk@yahoo.com

DOI

10.29121/granthaalayah.v10.i1.2022 4479

Funding: This research received no specific grant from any funding agency in the public, commercial, or not-for-profit sectors.

Copyright: (C) 2022 The Author(s). This is an open access article distributed under the terms of the Creative Commons Attribution License, which permits unrestricted use, distribution, and reproduction in any medium, provided the original author and source are credited.

\section{ABSTRACT}

Context: Provider-created demand has been found to be a component of out-of-pocket payments and consequent financial burden. Road traffic injuries are a classic case of medical uncertainty where treatment interventions could be induced especially when the provider's income is dependent on the quantity of care provided. The debate on the existence and form of SID continues unabated for the last three decades with no clear signs of a consensus. The paper argues that under conditions of uncertainty and imperfect competition in health care market, SID is quite likely and it is possible to detect. Methods: Three hundred non-fatal road traffic injury cases from seventeen hospitals (from public sector, private for-profit and not-for-profit sectors) were selected from three districts in Kerala, India. Besides, via case-study method appropriateness of treatment prescribed by the provider and utilized by the patient is evaluated. Using them, under-consumption or under-prescription of treatment or over treatment also assessed. Results \& Discussion: Firstly, higher level of service intensity was found in private sector compared to the public sector, even when the length of treatment for inpatients is higher in public hospitals. More than 76 percent of the patients who received less than 10 doses of medicines are in the public sector and 17 percent in private sector. Secondly, service intensity based on physician recommendation and use of scan across public and private providers finds that 52 percent of the entire scans were recommended by public sector. While 29 percent scans were prescribed by private health services and 19 percent by public and private sectors together. Thirdly, using a checklist and observation form, after controlling for co-morbidity and hospitals with similar facility with public and private ownership, two cases with clinically similar injury conditions were selected. While private health facilities were over- treating patients, public hospitals under-treat patients.

Keywords: Financial Burden, Road Traffic Injuries, Kerela

\section{INTRODUCTION}

Health care market displays some interesting but complex relationship between the care seeker and care giver. Due to the existence of asymmetry of information of differing degree, agency is both a solution and problem in costeffective utilization of health care. A solution because in the absence of agent, the principal (the care seeker) would have to spend a greater degree of time and energy to accumulate the knowledge for treating an ailment which is a remotely effective. A problem because imperfect principal - agent relationship directs the agent to work against the interest of principal, thereby increasing the cost and worsen quality of care. The second problem is generally described as supplier induced demand (SID) in health economics literature. Uncertainty in the outcome 
of health care interventions adds to the problem of costs and expenditure. Thus, more than the required care has implications for cost, access and burden.

In the context of information asymmetry and imperfections in the principal agent relationship, the provider/physician can shift the demand curve facing the patients which may benefit the provider at the cost of patients. This situation becomes prominent when the incentive structure facing the physician is fee-forservice and the number of health facilities and technologies available are beyond the normal requirements as reflected by the morbidity pattern. When patients consume more medical care than what is considered 'optimal' and prescriptions without any scientific justification led to what is called "flat-of-the-curve-medicine". Some researchers initially suspected the existence of SID because some of them had found that despite an increase in the supply of providers there is no reduction in the price of treatment Reinhardt (1985). Some also felt that the increases in the number of health care infrastructure (especially beds) was closely followed by increasing rate of utilization of beds Roemer (1961). Mooney (1994) argues that the physician reimbursement method of fee-for-service is associated with higher levels of provision. The debate on the existence and form of SID has been active in literature for the past four decades with no clear signs of a consensus. Different studies apply a variety of approaches to understand its existence, nature and magnitude. The validity of the results of various models is being debated. However, a discussion on these issues is beyond the objective of the present work.

Conceptual framework: We argue that the financial burden of treatment of households is assumed to be the function of health system and non-health system characteristics. Health system characteristics are defined as properties of actors and actions working towards the improvement of population health that get reflected through the four core functions of a health system called financing, provision, regulation and stewardship. How these four control knobs are being operationalised in a system is decisive in determining the financial burden faced by the individual's consequent upon an injury or illness. Conceptually, the paper argues that for the individual, under conditions of uncertainty cost of treating injuries and illnesses is not investment, rather it is a restorative or compensatory expenditure incurred as a consequence of a socially produced event called "road traffic injury".

One of the assumptions is that there is no major distinction between the output of the health care system (treatment) and outcome on the injured (health status). This assumption has become essential as there is a possibility that outcome of the treatment may differ between an injured having a history of better nourishment and otherwise as well as among different age groups. It is well-known that the outcomes of treatment may differ with age groups and the younger the age groups, the better the health outcome is and vice versa contributed by intrinsic biological reasons.

Injury care expenditure or the financial burden of the household of an injured can be captured using the following model:

FBT = f (pb, bp, a, g, y, cm, ls, p-a, ts, hp, ib, ins)

Where,

FBT Financial Burden or the total cost of the treatment

$\mathrm{pb}$ Severity of the injury (physical burden)

Bp Body part affected

a Age

s Gender

y Household income 
$\mathrm{cm}$ Co-morbidity

ls Length of stay

$\mathrm{p}-\mathrm{a}$ proposed minus actual treatment

ts Time space between proposed \& actual treatment

hp Health care provider

ib Institutional behaviour

ins Insurance

Various components of the total cost of injury care to the injured can be explained using the following expression

$\mathrm{C}=\mathrm{MC}+\mathrm{OC}(\mathrm{I})+\mathrm{OC}(\mathrm{H})+\mathrm{ML}+\mathrm{T}+\mathrm{INT}+\mathrm{M}$

Where,

C Cost of treatment

MC Medical Cost

OC(I) Opportunity cost of the injured

OC(H) Opportunity cost of the household

ML Medico-legal expenses

$\mathrm{T} \quad$ Cost of transport of the patient and bystanders

F Cost of food of the patient and bystanders

INT Interest and other costs of financing

M Miscellaneous (Vehicle damage, compensation, production and productivity loss to the society)

Medical cost can be further decomposed using the following equation:

$M C=f(c, s g, p h, d g, e q, s p)$

Where,

MC Medical cost of treating injury

c Consultation fees

sg Surgery

ph Medicines

dg Diagnostics

eq Equipment

sp Supplies

\section{METHODOLOGY}

Three hundred and two non-fatal road traffic injury cases from seventeen hospitals (from public sector, private for-profit and not-for-profit sectors) were selected from three districts in Kerala, India. Besides, via case-study method appropriateness of treatment prescribed by the provider and utilized by the patient is evaluated. Service intensity was measured using quantity of different medical services prescribed and used in the treatment process. Quantity of medicines and diagnostics recommended by providers were the indicators for inducement. With a specific type of injury, a panel of physician's general surgeons, orthopaedicians, and neurosurgeons serving both public and private sectors prepared a treatment protocol ranging from minimum essential to maximum appropriate prescriptions. 
Using them, under-consumption or under-prescription of treatment or over treatment also assessed.

The appropriateness of treatment prescribed by the provider and utilized by the patient is evaluated in the following way. For a specific case of injury, a panel of physicians from both public and private sector (including GP, Orthopeadician, General Surgeon and Neurosurgeon) were requested to prepare a treatment protocol ranging from minimum appropriate prescriptions to maximum appropriate prescriptions. Appropriate expenditures to take care of the prescription were calculated. In this under-consumption or under-prescription of treatment as well as over treatment were taken care of. While analyzing the treatment pattern and utilization, the setting (public/for-profit/not-for-profit) in which the care had been delivered as well as the level of health care provider was also taken note of.

\section{RESULTS}

\subsection{SERVICE INTENSITY ACROSS PROVIDERS}

Service intensity across different providers figure out that private sector has a higher level of service intensity when compared to the private sector, even when the length of treatment for inpatients is higher in public hospitals. Table 1 gives a brief idea regarding the intensity of medicine use (measured as total doses of medicines consumed per person) for the injured who had utilized the services of a private health unit, public health facility and at least one private and public health facility. More than 76 per cent of the patients who received less than 10 doses of medicines are in the public sector, and 17 percent in private sector. In rest of the rows, public as well as private sector shows more or less similar consumption levels. However, as given elsewhere, since the length of stay is longer in public hospitals and it could be expected to have a higher level of prescription. But the private sector, despite its relatively lower length of stay showed a higher intensity in medicine consumption.

\begin{tabular}{|c|c|c|c|c|}
\hline \multicolumn{5}{|c|}{ Table 1 Proportion of Medicine Prescription Across Providers } \\
\hline $\begin{array}{l}\text { Medicines } \\
\text { (Quantity) }\end{array}$ & Public & Private & Public \& Private & Total \\
\hline$<10$ & $67(76.1)$ & $15(17.0)$ & $6(6.8)$ & $88(33.8)$ \\
\hline $10-25$ & $15(45.5)$ & $15(45.5)$ & $3(9.1)$ & 33 (12.7) \\
\hline $26-50$ & $22(40.0)$ & $15(27.3)$ & $18(32.7)$ & $55(21.2)$ \\
\hline $51-100$ & $8(19.5)$ & $10(24.4)$ & $23(56.1)$ & $41(15.8)$ \\
\hline$>100$ & $22(51.2)$ & $12(27.9)$ & $9(20.9)$ & $43(16.5)$ \\
\hline Total & $134(51.5)$ & 67 (25.8) & 62 (22.7) & $260(100.0)$ \\
\hline
\end{tabular}

Analysis of service intensity based on the recommendation and use of scan across public and private providers finds that 51.8 per cent of the entire scans were recommended by public sector (Table 2 ). While 29.1 per cent scans were prescribed by private health services and 19 per cent by public and private sectors together. However, when broken up, the fraction of cases where more than one scan is recommended and used was in the private sector. For example, more than 55 per cent of the cases who had more than one scan are in the private sector followed by public and private sector together. 


\begin{tabular}{|ccccc|}
\hline Table 2 Proportion of Scan Prescription Across Providers \\
\hline \multicolumn{5}{c}{ NUMBER OF THE INJURED } \\
\hline Scan (Number) & Public & Private & Public \& Private & Total \\
\hline 1 & $54(49.1)$ & $21(19.1)$ & $15(13.6)$ & $90(81.8)$ \\
2 & $3(5.3)$ & $11(10.0)$ & $6(5.5)$ & $20(18.2)$ \\
Total & $57(51.8)$ & $32(29.1)$ & $21(19.1)$ & $110(100.0)$ \\
\hline
\end{tabular}

Participant observation - a case study

This is the result of the analysis undertaken using a protocol with the help of the tool - "Participant Observation Form". One patient each with head injury (forehead) was taken to a hospital A (a private hospital) and (hospital B) a public hospital respectively. Both the hospitals are referral centres and as such provide advanced care. One hospital with a bed occupancy rate of more than 170 per cent and another with a bed utilization rate close to fifty per cent.

Clinical condition of both the patients are:

Blood pressure: Normal

Pulse rate. Normal

Loss of consciousness: No

Physical examination: Wound on the skull and no other external injury

History of vomiting: No

ENT Blooding: Nil

Glasgow Coma Scale (GCS): 15/15

\section{Interventions}

In hospital A, the injured was admitted for two days and was advised a CT scan and medicines and wound debridement.

In hospital B, the patient was advised observation for a period of five hours and advised routine medicines. Wound debridement was undertaken and a CT was advised, only if GCS worsens, which has not happened either.

\section{Clinical assessment}

Hospital A advised a CT scan to the patient without any medically justified reason, because objectives conditions like no history of vomiting, no ENT blooding, adequate GCS were found. There is also no apparent reason for admission and the patient could have been treated as out-patient with some extended hours of observation.

Hospital B has gone almost close to the criteria of medically justified interventions. However, there was a delay in getting treatment at fast speed, and the crowded state of the observation room probably led the physician to prescribe five hours of observation which could be extended for about 10 hours.

Incentives faced by the provider constitute the gold-mark for distinguishing between inefficiency at provider level and supplier induced demand. In many economic studies dealing with the health care sector the physician has an informational advantage in supplying medical services Liu and Mills (1999). The existence of incentives is the kingpin in supplier-induced demand and any incentive structure facing the provider where the revenue of the provider has a direct linkage with the quantity of services provided, and then one could unambiguously doubt a clear chance for inducing demand. In a study, Muraleedharan finds prima facie to 
suspect the existence of supplier-induced demand of diagnostic services in Chennai, where the physicians have mostly accepted that there exists networking between private medical institutions and diagnostic firms Muraleedharan (1999). Another study found that there existed wide variations in the prescription of drugs for the same health condition with 75 percent of the consumption was ruled unnecessary by an expert panel Meng et al. (2000).

\section{DISCUSSION}

Over-prescription is one of the prime reasons for injured downgrading the ranking of private sector, as found in the study. Some studies also reached similar findings elsewhere. For instance, the private sector health facilities were less likely to refer the patients to higher levels of care Meng et al. (2000). The private facilities try to retain the patient till some serious complications arise, and such referrals would be towards public sector so that there is an absolvement of responsibility. The evasion is because of two things: Firstly, when dealing with patients with serious complications, there is a higher probability of risk involved which can be safely shifted to the public sector or another higher-level hospital. Secondly, death to a patient also means the reputation of the hospital is at stake which will make the facility lose some proportion of the existing or potential market.

The phenomenon that availability leading to utilization is more or less clearly established in the analysis. Firstly, hospitals with lower utilization (measured by lower bed occupancy rate) tend to have a longer length of stay than a hospital with higher bed occupancy rate in public and private sectors at different levels, presuming severity remains same. Secondly, there exists a positive correlation between hospitals having availability of scans and utilization of such facilities across different providers, assuming severity is same across providers.

The present study found that diagnostic firms reported paying 'rewards' to physicians who refer patients to their diagnostic firms. Diagnostic firms working independently charge the highest across the study site. These firms have tie-ups with many private hospitals and public physicians. Cases which require complex analysis are referred to some diagnostic facility within a public hospital. One of the physician interviews revealed that majority of the scan results reported from these private diagnostic firms have results 'no relevant findings' further confirming the patient being subjected to additional financial burden of injury care. The study supports the view of a scholar who has argued earlier that competition in medical care sometimes led to 'flat-of-the- curve medicine' Newhouse (1982). Existence of SID in a system starts a vicious cycle of cost escalation and ineffective treatment. For once a patient is prescribed more than necessary, the later on patients are also likely to be prescribed more interventions and it becomes a practical standard later on. Majority of the providers reported that there are not much review mechanisms in place to ensure follow up of standards. As seen elsewhere, medical care market in the State has grown without proper policy governing its location, quality of services, price, functioning requirements, access to the unreached etc. The regulatory mechanisms in place lack adequate power or non-existent and existing system have huge number of rent seekers. The policy implications include; changing the incentive structure faced by the provider, utilization reviews, regulating the supply etc so that loss of welfare indicated by consumer surplus can be reduced. The central limitation the study encountered is that the share of SID should have been calculated using a method which incorporates the quantity of excess prescriptions multiplied by their market price would have offered a reasonable estimate of financial burden. 


\section{SUMMARY}

In health care markets where the providers are reimbursed on the basis of quantity of interventions prescribed and utilised, providers have a tough incentive to induce more interventions than are medically justified. Thus, SID has implications for cost, access and burden. The study finds the existence of SID though does not exactly quantify the actual volume of financial burden imposed by SID. The medical care market in the State has grown without proper policy governing its location, quality of services, price, functioning requirements, access to the unreached etc. A detailed analysis of the medical sub-markets is essential to understand the pricing behaviour in the market.

\section{REFERENCES}

Liu X \& Mills A (1999) Evaluating payment mechanisms: how can we measure unnecessary care? Health Policy and Planning ; 14 (4) :409-13. Retrieved from https://doi.org/10.1093/heapol/14.4.409

Meng QY, Liu X \& Shi Junshi (2000) Comparing the services and quality of private and public clinics in rural China. Health Policy and Planning 15 (4) : 349356. Retrieved from https://doi.org/10.1093/heapol/15.4.349

Mooney GH (1994) Key issues in Health Economics, Hemel Hempstead: Harvester Wheatsheaf.

Muraleedharan, VR (1999) Characteristics and structure of the private hospital sector in urban India : a study of Madras city. Small Applied Research Paper No. 5, Partnership for Health Reform Project, Abt. Associates, Bethesda, USA.

Newhouse JP (1982). Is competition the answer? Journal of Health Economics 1 : 109-116. Retrieved from https://doi.org/10.1016/0167-6296(82)90023-6

Reinhardt (1985). "The theory of Physician-Induced Demand: Reflections after a decade." Journal of Health Economics 4 (Summer) :187-93. Retrieved from https://doi.org/10.1016/0167-6296(85)90008-6

Roemer, MI (1961) Bed supply and hospital utilization: à natural experiment. Hospitals 1 November : 35 - 42 